\title{
Single-incision Laparoscopic Surgery for Simultaneous Appendectomy and Cholecystectomy
}

\author{
Sang-Hong Choi, M.D., Seung-Hyun Lee, M.D., Byung-Kwon Ahn, M.D., Sung-Uhn Baek, M.D. \\ Department of Surgery, Kosin University College of Medicine, Busan, Korea
}

Single-incision laparoscopic surgery (SILS) has become popular due to the advantage of minimizing surgical. We report on two cases of simultaneous appendectomy and cholecystectomy using a single-incision laparoscopic technique.

The patients were 49- and 50-year old females diagnosed with acute appendicitis with concomitant cholelithiasis. Body mass indices of the patients were 22.3 and 26.0 . A $3 \mathrm{~cm}$ abdominal incision was made via the umbilicus, and a single port platform was created using a small wound retractor $\left(\right.$ ALEXIS $^{\circledR}$ wound retractor S, Applied Medical, Santa Margarita, CA, USA) and a surgical glove. Cholecystectomy was per- formed first, followed by the appendectomy. The operation times were 165 and 280 minutes, and blood loss was 50 and $150 \mathrm{cc}$, respectively. The postoperative hospital stays were five and seven days, and one patient had a wound seroma as a surgical complication. We believe that SILS for simultaneous appendectomy and cholecystectomy is a feasible and safe minimally invasive procedure.

Key words: Single-incision laparoscopic surgery, Appendectomy, Cholecystectomy

\section{INSTRUCTION}

Single-incision laparoscopic surgery (SILS) is an innovative surgical procedure performed via a single umbilical laparoscopic incision compared with the several abdominal trocar sites required during conventional laparoscopic surgery. Recently, SILS has become more popular due to the advantage of minimizing surgical trauma for a wide variety of surgical procedures in the fields of general surgery, urology, and gynecology. ${ }^{1}$ Although many studies have showed that SILS is a feasible, safe procedure, it is technically challenging compared with conventional laparoscopic surgery.

Appendectomy and cholecystectomy are two of the most frequently performed operations to utilize SILS. ${ }^{2-4}$ However, there are no reported cases of simultaneous appendectomy and cholecystectomy using a single-incision laparoscopic technique. Herein, we report two cases of simultaneous appendectomy and cholecystectomy using the single-incision laparoscopic technique.

Received May 18, 2014, Revised August 5, 2014,

Accepted August 19, 2014

※ Corresponding author : Seung-Hyun Lee

Department of Surgery, Kosin University College of Medicine, 34 Amnam-dong, Seo-gu, Busan 602-702, Korea

Tel : +82-51-990-6462, Fax : +82-51-246-6093

E-mail : gscrslsh@ $@$ hanmail.net

\section{CASE REPORTS}

\section{1) Case 1}

A 49-year-old female patient presented to the emergency department with a chief complaint of right lower quadrant abdominal pain. Two days prior to admission, she experienced epigastric discomfort, which did not improve after taking digestive medicine. She reported a shifting pain to the right lower abdomen quadrant on the day of admission. She also complained of intermittent nausea but denied vomiting. She had intermittent epigastric and right upper quadrant abdominal pain. She had no history of previous abdominal surgery.

On admission, the patient's height, weight, and body mass index were $156 \mathrm{~cm}, 57 \mathrm{~kg}$, and 22.3, respectively. Vital signs were normal with blood pressure of $100 / 90 \mathrm{mmHg}$, pulse rate of 72 beats/min, respiration rate of 20/min, and body temperature of $37.4^{\circ} \mathrm{C}$. Physical examination revealed tenderness and rigidity in the right lower abdominal quadrant. Intestinal sounds were audible on auscultation but were weak with decreased frequency. Laboratory investigations revealed the following:

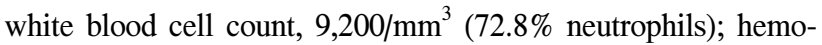
globin, $9.8 \mathrm{~g} / \mathrm{dl}$; hematocrit, $30.4 \%$; platelet count, $278 \times 10^{3}$ / $\mathrm{mm}^{3}$; serum protein, $6.6 \mathrm{~g} / \mathrm{dl}$; serum albumin $3.7 \mathrm{~g} / \mathrm{dl}$; total bilirubin, $0.3 \mathrm{mg} / \mathrm{dl}$; alanine aminotransferase $17 \mathrm{IU} / \mathrm{l}$; aspartate aminotransferase, $13 \mathrm{IU} / 1$; alkaline phosphatate, $51 \mathrm{U} / \mathrm{L}$; amylase, $51 \mathrm{U} / \mathrm{L}$; lipase, $34 \mathrm{U} / \mathrm{L}$; creatinine, $0.5 \mathrm{mg} / \mathrm{dl}$; serum $\mathrm{Na}$, $139 \mathrm{meq} / \mathrm{l}$; serum K, $3.7 \mathrm{meq} / \mathrm{l}$. 

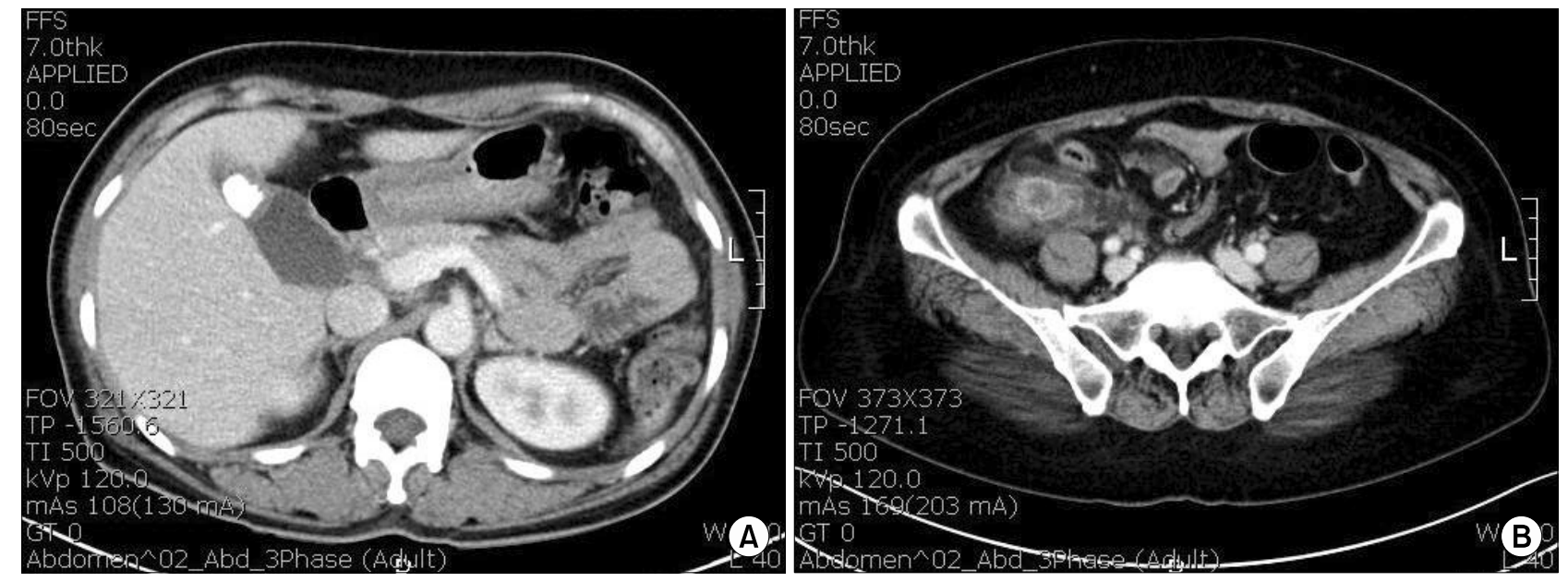

Fig. 1. Computed tomography revealed about $1.7 \mathrm{~cm}$ sized stone in the gall bladder. The wall of the gall bladder was slightly thickened (A). Suspicious thickening of the wall of the appendix was also identified (B).

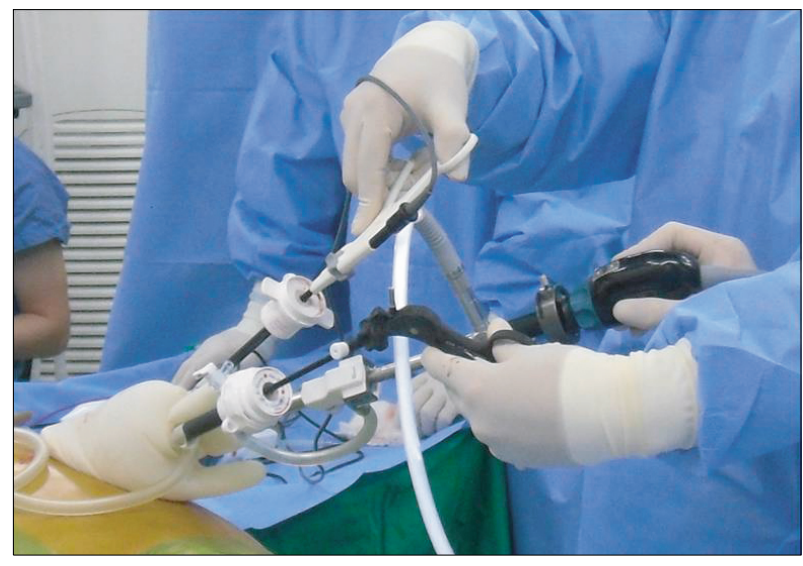

Fig. 2. Homemade single-port platform.

Computed tomography revealed an approximately $1.7 \mathrm{~cm}$ stone in the gall bladder and a slightly thickened gall bladder wall. Suspicious thickening of the wall of the appendix was also identified (Fig. 1A and 1B). A presumptive diagnosis of acute appendicitis with concomitant cholelithiasis was made. On the day of admission, the patient underwent emergent surgery and elected to undergo simultaneous cholecystectomy. After counseling the patient extensively on the various laparoscopic techniques, she gave consent for a simultaneous appendectomy and cholecystectomy with the single-incision laparoscopic technique.

With the patient under general anesthesia, a $3.0-\mathrm{cm}$ umbilical vertical skin incision and a $2.0-\mathrm{cm}$ fasciotomy were made to insert a wound retractor (ALEXIS ${ }^{\circledR}$ wound retractor S, Applied Medical, Santa Margarita, CA, USA). A surgical glove was ap- plied to the wound retractor with a $5 \mathrm{~mm}$ trocar on first finger tip, a $10 \mathrm{~mm}$ trocar on third finger tip, and a $10 \mathrm{~mm}$ trocar on fifth finger tip. Pneumoperitoneum was created with $\mathrm{CO} 2$ gas insufflation, and abdominal pressure was maintained at $10 \sim$ $12 \mathrm{mmHg}$. Surgery was performed by inserting a $10 \mathrm{~mm}, 0$ degree laparoscopic camera through the trocar of the third finger and inserting the surgical instruments through the remaining two trocars (Fig. 2).

Cholecystectomy was performed first and in a standard manner. The gall bladder was placed into an Endo-Catch bag and relocated to the right upper quadrant of the abdomen. Appendectomy was performed after the cholecystectomy was completed. The base of the appendix was tied two times with an endo-loop, and two graspers were used to retrieve the appendix and Endo-Catch bag containing the gall bladder. The surgical glove was then separated from the wound retractor to retrieve the specimens. The operation time was 165 minutes and blood loss was $50 \mathrm{cc}$. A liquid diet was started on postoperative day 2 , and the total hospital stay was 5 days. The postoperative recovery was uneventful (Table 1).

\section{2) Case 2}

A 50-year-old female patient presented to the emergency department with a chief complaint of right lower abdominal quadrant pain that developed 5 days prior. She visited a local medical clinic and was diagnosed with acute appendicitis after an ultrasound examination. She had intermittent epigastric and right upper quadrant abdominal pain. She had no history of previous abdominal surgery.

On admission, the patient's height, weight, and body mass 
Table 1. Single-incision laparoscopic surgery for simultaneous appendectomy and cholecystectomy

\begin{tabular}{|c|c|c|}
\hline & Case 1 & Case 2 \\
\hline Sex & Female & Female \\
\hline Age (years) & 49 & 50 \\
\hline BMI & 22.3 & 26.0 \\
\hline ASA score & 1 & 1 \\
\hline Diagnosis & $\begin{array}{l}\text { Appendicitis, } \\
\text { concomitant } \\
\text { cholelithiasis }\end{array}$ & $\begin{array}{l}\text { Appendicitis, } \\
\text { concomitant } \\
\text { cholelithiasis }\end{array}$ \\
\hline Operation time $(\mathrm{min})$ & 165 & 280 \\
\hline Blood loss (cc) & 50 & 150 \\
\hline Diet (day) & 2 & 4 \\
\hline $\begin{array}{l}\text { Postoperative hospital stay } \\
\text { (day) }\end{array}$ & 5 & 7 \\
\hline Postoperative complication & None & Wound seroma \\
\hline
\end{tabular}

index were $158 \mathrm{~cm}, 65.3 \mathrm{~kg}$, and 26.0, respectively. Vital signs were normal with a blood pressure of $120 / 90 \mathrm{mmHg}$, pulse rate of 68 beats/min, respiration rate of 20/min, and body temperature of $36.7^{\circ} \mathrm{C}$. Physical examination revealed tenderness and rigidity of the right lower abdominal quadrant. Laboratory investigations revealed the following: white blood cell count, $11,400 / \mathrm{mm}^{3}$ (62.4\% neutrophils); hemoglobin, $12.7 \mathrm{~g} / \mathrm{dl}$; hematocrit, $36.4 \%$; platelet count, $242 \times 10^{3} / \mathrm{mm}^{3}$; total bilirubin, 0.9 $\mathrm{mg} / \mathrm{dl}$; alanine aminotranferase, $38 \mathrm{IU} / 1$; aspartate aminotransferase, $41 \mathrm{IU} / \mathrm{l}$; alkaline phosphatate, $139 \mathrm{U} / \mathrm{L}$; amylase, 68 $\mathrm{U} / \mathrm{L}$; lipase, $48 \mathrm{U} / \mathrm{L}$; creatinine, $0.5 \mathrm{mg} / \mathrm{dl}$; serum Na, 141 meq/1; serum K, $3.9 \mathrm{meq} / \mathrm{l}$.

Computed tomography revealed several stones in the gall bladder, slightly thickened gall bladder wall, and thickening of the appendix wall. A presumptive diagnosis of acute appendicitis with concomitant cholelithiasis was then made. On the day of admission, the patient underwent emergent surgery using the same technique as in Case 1. The total operation time was 280 minutes with a blood loss of $150 \mathrm{cc}$. Liquid diet was started on postoperative day 4 , and the total hospital stay length was 7 days. The patient suffered a wound seroma as the only postoperative complication (Table 1).

\section{DISCUSSION}

SILS represents an innovative technique of minimally invasive surgery. The advantages of SILS include a reduction in the number of incisions, improved cosmetic results, decreased postoperative pain, fewer wound-related complications, and decreased hospital stay. An apparent advantage of SILS is the ex- cellent cosmetic results through an intra-umbilical or a sub-umbilical incision of $1.5 \sim 2.5 \mathrm{~cm}$ in length. However, SILS has intra-operative procedure-related challenges, including instrument crowding and crossover, limited view, and inadequate retraction. These challenges may be related to higher rates of intra-operative and postoperative complications. ${ }^{5}$

Recently, in spite of the intra-operative procedure-related challenges, SILS has been used for a variety of general, urologic, and gynecologic surgeries. ${ }^{1-3}$ Appendectomy and cholecystectomy are two of the most frequently performed procedures to utilize SILS. ${ }^{2-4}$ For the efficacy and safety of SILS in appendectomy compared to conventional laparoscopic surgery, Gao et al. $^{6}$ reported results of a meta-analysis. The mean operative time was found to be significantly longer in SILS compared to conventional laparoscopic surgery. There were no significant differences in visual analogue scale (VAS) pain scores, wound infection rates, overall complications, or postoperative hospital stays. They concluded that SILS represents a possible alternative to conventional laparoscopic surgery for appendectomy. For cholecystectomy, Markar et al. ${ }^{7}$ reviewed seven randomized controlled trials containing a total of 195 cholecystectomies using SILS and 180 conventional laparoscopic surgeries. The study showed that the operative time was significantly longer in the SILS group, but no significant differences were found between the groups in terms of postoperative complication incidence, postoperative pain scores (VAS), or length of hospital stay. They concluded that SILS cholecystectomy is a safe procedure for the treatment of uncomplicated gallstone disease.

For SILS, an intra-umbilical or sub-umbilical incision is used as the initial port placement to perform a variety of general surgery, urologic, and gynecologic procedures. This umbilical incision site can be used to perform multiple concomitant procedures. In the literature, a few simultaneous procedures using SILS have been reported, such as hysterectomy and appendectomy, bilateral nephrectomy, cholecystectomy and ovary cystectomy, cholecystectomy and hysterectomy, as well as herniorrhaphy and nephrectomy. ${ }^{8-10}$

However, there are no reports of simultaneous appendectomy and cholecystectomy using the single-incision laparoscopic technique. In this report, two cases of simultaneous appendectomy and cholecystectomy were performed for appendicitis with uncomplicated cholelithiasis by one surgeon. For the operative platform, a single-port setting was created using a small wound retractor (ALEXIS ${ }^{\circledR}$ wound retractor S, Applied Medical, Santa Margarita, CA, USA) and a surgical glove. The operation time was not short (165 and 280 mins). The case 1 was the 
first case of single-port surgery for the surgeon. The long operation time is considered to relate with the learning curve for single port surgery. However, reduction in the number of port placements and small single wound may be considered as its advantages. We believe that SILS for simultaneous appendectomy and cholecystectomy is a feasible and safe minimally invasive procedure.

\section{REFERENCES}

1) Ahmed K, Wang TT, Patel VM, et al. The role of singleincision laparoscopic surgery in abdominal and pelvic surgery: a systematic review. Surg Endosc 2011;25:378-396.

2) Chow A, Purkayastha S, Paraskeva P. Appendicectomy and cholecystectomy using single-incision laparoscopic surgery (SILS): the first UK experience. Surg Innov 2009;16:211-217.

3) Hong TH, You YK, Lee KH. Transumbilical single-port laparoscopic cholecystectomy: scarless cholecystectomy. Surg Endosc 2009;23:1,393-1,397.

4) Chouillard E, Dache A, Torcivia A, et al. Single-incision laparoscopic appendectomy for acute appendicitis: a prelim- inary experience. Surg Endosc 2010;24:1,861-1,865.

5) Rehman H, Ahmed I. Technical approaches to single port/ incision laparoscopic appendectomy: a literature review. Ann R Coll Surg Engl 2011;93:508-513.

6) Gao J, Li P, Li Q, Tang D, Wang DR. Comparison between single-incision and conventional three-port laparoscopic appendectomy: a meta-analysis from eight RCTs. Int J Colorectal Dis 2013;28:1,319-1,327.

7) Markar SR, Karthikesalingam A, Thrumurthy S, Muirhead L, Kinross J, Paraskeva P. Single-incision laparoscopic surgery (SILS) vs. conventional multiport cholecystectomy: systematic review and meta-analysis. Surg Endosc 2012;26:1205-1213.

8) Page T, Soomro NA. Bilateral simultaneous single-port (LESS) laparoscopic nephrectomy (laparoendoscopic single site surgery). Indian J Urol 2010 Oct;26(4):590-592.

9) Surico D, Gentilli S, Vigone A, Paulli E, Leo L, Surico N. Laparoendoscopic single-site surgery for treatment of concomitant ovarian cystectomy and cholecystectomy. J Minim Invasive Gynecol 2010;17:656-659.

10) Hart S, Ross S, Rosemurgy A. Laparoscopic single-site combined cholecystectomy and hysterectomy. J Minim Invasive Gynecol 2010;17:798-801. 